anales de psicología, 2018, vol. 34, $\mathrm{n}^{\circ} 2$ (mav), 264-273 http://dx.doi.org/10.6018/analesps.34.2.289271
(C) Copvright 2018: Editum. Servicio de Publicaciones de la Universidad de Murcia. Murcia (Spain) ISSN print edition: 0212-9728. ISSN web edition (http://revistas.um.es/analesps): 1695-2294

\title{
Five-factor model of personality disorders: Spanish normative data and validation
}

\author{
Joaquín Colodro $^{1 *}$, Juan J. López-García ${ }^{1}$, Laura Mezquita ${ }^{2,4}$, Lucía Colodro-Conde ${ }^{1,3}$, Manuel I. Ibáñez ${ }^{2,4}$, \\ Silvia Edo ${ }^{2,5}$, Elena Villa ${ }^{2}$, and Generós Ortet ${ }^{2,4}$ \\ 1 Universidad de Murcia (Spain). \\ 2 Universitat Jaume I (Spain). \\ 3 QIMR Berghofer Medical Research Institute (Australia). \\ 4 Centro de Investigación Biomédica en Red de Salud Mental (CIBERSAM), Instituto de Salud Carlos III, Madrid (Spain). \\ 5 Hospital de Día, Hospital Provincial de Castellón (Spain).
}

\begin{abstract}
Título: Modelo de los cinco factores de los trastornos de personalidad: Baremo español y validación.

Resumen: La concepción categórica de los trastornos de personalidad (TP) ha dado paso al paradigma dimensional, donde el modelo de los Cinco Factores (MCF) propone hipótesis teóricas para describir la patología de la personalidad y prototipos empíricos de los TP del DSM, además de técnicas para valorarlos en base a facetas del NEO PI-R. En este estudio ex post-facto se han elaborado baremos para el recuento de TP-MCF a partir de la adaptación española del NEO PI-R. Además, se ha comprobado la coherencia diagnóstica con IPDE y la validez de los recuentos de TP-MCF en una muestra clínica $(n=222)$ y otra no clínica $(n=742)$. A partir de las puntuaciones en NEO PI-R se elaboró el baremo español de los TP-MCF, cuyas cotas significativas son superadas con elevada probabilidad por casos subclínicos detectados con IPDE. Las correlaciones convergentes entre los recuentos de TP-MCF y los equivalentes casos de TP-DSM fueron estadísticamente significativas y superaron a cualquier correlación divergente y a la correlación divergente media en todos los TP-MCF. El recuento de facetas relevantes en TP-MCF y el baremo español resultante facilitan la comprensión e interpretación de los TP en distintos ámbitos de la psicología aplicada.

Palabras clave: MCF; trastorno de personalidad; NEO PI-R; baremo es-
\end{abstract} pañol; validez de constructo.

\section{Introduction}

Historically, personality disorders (PD) have been considered peculiar forms of mental illness and qualitatively distinct clinical syndromes. The categorical approach of PDs in the Diagnostic and Statistical Manual of Mental Disorders (DSM) derived from empirical data of clinical practice and psychiatric research has advantages in terms of easy communication and diagnostics. However, failing to consider a model of personality in its formulation results in diagnostic criteria with significant limitations in their theoretical foundation (Clark, 2007; Morey, Benson, Busch, \& Skodol, 2015; Trull \& Durrett, 2005; Widiger \& Simonsen, 2005; Widiger \& Trull, 2007). Their categorical classification remains in DSM, although DSM-5 includes a dimensional alternative (American Psychiatric Association, 2000, 2013).

Given these difficulties, dimensional models redefined PDs based on a model of personality traits unrelated to any traditional diagnostic system (Morey et al., 2007; Trull \& Durrett, 2005; Widiger, 2007; Widiger \& Simonsen, 2005). A possible dimensional model of PDs is the five-factor model (FFM) of personality (Trull, 2012; Widiger, 2015; Widiger \&

* Correspondence address [Dirección para correspondencia]: Joaquín Colodro Plaza, GI E038-06 Psicología del Deporte, Universidad de Murcia, Campus de Espinardo, 30100 Murcia (Spain).

E-mail: jcplaza@um.es
Abstract: The categorical approach of personality disorders (PD) has given way to a dimensional paradigm. Within this, the Five-factor model (FFM) proposes theoretical hypotheses describing personality pathologies and PD empirical prototypes based on the DSM (DSM-PD). Moreover, a methodology to score DSM-PD using the NEO PI-R facets was developed. In this ex post-facto study FFM-PD count norms were developed using data from the NEO PI-R Spanish adaptation. Furthermore, the diagnostic agreement with the IPDE and validity of FFM-PD counts was analyzed in a clinical $(n=222)$ and non-clinical sample $(n=742)$. Based on NEO PI-R scores, we presented Spanish FFM-PD normative data. FFMPD benchmarks were highly likely to be exceeded if subjects were classified as a subclinical case in the DSM-PD. Convergent correlations of FFM-PD counts with their equivalent subclinical cases of DSM-PD were statistically significant and outperformed any divergent correlation as well as the average divergent correlations in all FFM-PD. The use of a count technique based on NEO PI-R facets and Spanish FFM-PD normative data facilitate PD understanding and interpretation in various applied psychology fields.

Keywords: FFM; personality disorder; NEO PI-R; Spanish normative data; construct validity.

Costa, 2013; Widiger \& Trull, 2007). The FFM traits, in addition to being relatively stable, endogenous and substantially genetic based entities, have explanatory power of personality and behavior (John, Naumann, \& Soto, 2008; McCrae \& Costa, 1997). The FFM, as assessed by the NEO Personality Inventory-Revised (NEO PI-R), consists of five bipolar dimensions with six facets each, having differentiated poles as maladaptive extreme variants which impact daily life (Bagby, Costa, Widiger, Ryder, \& Marshall, 2005; McCrae, Löckenhoff, \& Costa, 2005; Mullins-Sweatt \& Widiger, 2010; Widiger \& Costa, 2013). The FFM can be used for an inclusive classification of the normal and abnormal personality functioning (Miller, Lynam, Widiger, \& Leukefeld, 2001; Nestadt et al., 2008; Widiger \& Costa, 2012, 2013; Widiger \& Mullins-Sweatt, 2009). The FFM allows development of a specific profile for each PD with theoretical significance and diagnostic capability in terms of dimensions and facets assessed with the NEO PI-R (Mullins-Sweatt \& Widiger, 2006; Widiger \& Costa, 2012).

The first hypotheses about FFM capacity to represent personality pathology emerged from a theoretical attempt to describe the PDs of DSM-IV (DSM-PD), encoding its diagnostic criteria on the basis of the high, low, or neutral levels of FFM facets in each PD (Widiger \& Costa, 2013; Widiger, Trull, Clarkin, Sanderson, \& Costa, 1994, 2002). From an empirical viewpoint, PD prototypes were developed in the research and clinical practice fields (Lynam \& Widiger, 2001; 
Samuel \& Widiger, 2004; Widiger \& Costa, 2012) and these proposals were used as hypothesis in subsequent empirical studies. A meaningful line of research on the relationship between FFM and PD has been developed since the beginning of the century (Widiger \& Costa, 2013). Traditional reviews (Clark, 2007), factor analysis of published data (O'Connor, 2005) and meta-analysis (Samuel \& Widiger, 2008; Saulsman $\&$ Page, 2004) led to the conclusion that strong relationships exist between DSM-PD conception and normal personality traits, enabling a characterization of each PD with a specific FFM profile. Accordingly, different scoring techniques have been proposed to compare profiles produced with PD facets in the FFM (FFM-PD) with NEO PI-R scores: a profile comparing technique which matches a personality profile to every PD prototype and a simpler additive count technique only requiring accumulation of relevant facet scores for each prototype. FFM-PD counts represent a suitable method for PD estimation, proving a high degree of concordance with the prototype-matching technique (Lynam \& Widiger, 2001; Miller, Bagby, Pilkonis, Reynolds, \& Lynam, 2005; Samuel \& Widiger, 2004, 2008; Widiger \& Mullins-Sweatt, 2010). Both procedures for scoring individuals' FFM data, however, provide results difficult to interpret directly without contextualizing individual results with normative data. The first published normative data on NEO PI-R for FFM-PD counts were obtained in North American, French, and BelgianDutch samples. The diagnostic efficiency of the count method was originally tested in clinical samples using the $T \geq 65$ cutoff point to identify people who met DSM-PD criteria (Miller et al., 2008).

It is very important to identify PDs as these relate to functional impairment and reduced quality of life similarly to other mental disorders, and in addition they have specific social problems (American Psychiatric Association, 2013; Hengartner, Müller, Rodgers, Rössler, \& Ajdacic-Gross, 2014). However, their diagnosis is not easy or solved. In practice, the clinician's experience is basically used to detect and identify PDs, while self-report questionnaires are used for research purposes to guide diagnostic work and reduce global semi-structured interview duration (Widiger \& Boyd, 2009). The degree of agreement between PD categorical diagnoses carried out with different instruments is not satisfactory (Nestadt et al., 2012; Samuel, 2015). Dimensional models can provide a solution to improve PD predictive validity with the count approach of salient facets of NEO PI-R (Lynam, 2012). This procedure can be valid for the last two versions of DSM, because identical categories and criteria from DSM-IV for the PDs are in Sect. II of DSM-5 and similar constructs in Sect. III (Miller, Few, Lynam, \& MacKillop, 2015; Morey \& Skodol, 2013; Widiger, 2015). Given the lack of normative data on the Spanish population, the consolidation of DSM-PD prototypes and techniques to score PDs from the FFM encourages the development of specific normative data and benchmarks for estimating PDs.

Therefore, the objective of this ex post facto study is twofold: (a) to develop Spanish normative data valid to de- scribe and interpret FFM-PD counts from the NEO PI-R facets; and (b) to test FFM-PD normative data validity in Spanish samples so as to describe and analyze PD symptom counts assessed with a specific screening questionnaire.

\section{Methods}

\section{Participants}

The authors of the NEO PI-R Spanish adaptation used a normative sample of 1000 men and 1000 women, selected from a heterogeneous sample $(n=26,972)$ who underwent selection processes (Costa \& McCrae, 2008). The norms of the FFM-PD counts for each sex and total sample were developed with the data from this sample.

To check normative data validity, we applied a nonclinical sample (Sample 1) of 742 participants (63.5\% women, age $M=23.25, S D=3.71)$, mostly students $(69.5 \%)$ with $19.4 \%$ active workers, $7.3 \%$ unemployed, and $3.8 \%$ others. $63.2 \%$ had an income lower than $€ 450,16.6 \%$ between $€ 450$ $900,12.9 \%$ between $€ 900-1500$ and the $7.3 \%$ had an income higher than $€ 1500.67 .5 \%$ lived with their parents, $10.4 \%$ with their partner, $4.6 \%$ alone and $17.5 \%$ others (e.g., housemate). In addition, we used a second clinical sample (Sample 2) composed of 222 patients (65.77\% women) treated at an outpatients' service and with a mean age of 36.66 $(S D=10.90$; range $=17$ to 63$)$. A total of $37 \%$ of these patients had primary studies, $35 \%$ secondary studies, $26 \%$ university studies and the remaining $2 \%$ no studies.

\section{Instruments}

International Personality Disorder Examination (IPDE), Module DSM-IV. The Spanish version (López-Ibor, Pérez-Urdaniz, \& Rubio, 1996) of the IPDE (Loranger, 1995) was used. Its 77 items of true/false answers are intended to identify all 10 PDs covered in the DSM-IV and retained in the DSM-5 (American Psychiatric Association, 2000, 2013). The existence of a possible PD is linked to a score of three or more symptoms, such as the subclinical case criterion. According to the manual, reliability and validity data indicate that IPDE is a suitable research instrument. Studies with non-clinical samples show a sensitivity rate of detection on the order of $100 \%$ and a specificity of $61 \%$. Internal consistency coefficients showed inadequate reliability coefficients in Sample 1 and 2.

Revised NEO Personality Inventory (NEO PI-R). An assessment of the adult personality in accordance with the FFM is obtained with the Spanish adaptation of the original Costa and McCrae questionnaire (2008), comprising 240 items which are answered according to a 5 -point Likert scale and which provides scores for five broad dimensions and their thirty facets. The internal consistency coefficients in the normative Spanish sample for dimensions $(.85>\alpha \leq .91)$ and facets $(55>\alpha \leq .81)$ is rendered acceptable and similar to those of the USA version. In our study these coefficients 
showed a similar dimension range $(.86>\alpha \leq .92$ in Sample 1 and $.83>\alpha \leq .90$ in Sample 2). Although Sample 1 and 2 had facets with two and four low values $(<.50)$, the magnitude of median coefficients $(M d n=.71$ and .72 , respectively) was similar to that of the Spanish normative sample.

FFM-PD counts. The FFM-PD counts is a simplified technique proposed by Miller et al. (2005) to make estimations based on relevant NEO PI-R facets and their high or low contribution to each DSM-PD prototype, including the revised count of Dependent PD (Lynam \& Widiger, 2001; Miller \& Lynam, 2008). See the information on calculation technique for the every FFM-PD count in Appendix. Similarly to previous studies, we used a criterion of $T \geq 65$ cutoff point to detect a possible PD and indicate the need for additional assessment (Miller, 2012; Miller et al., 2008).

In addition to the previous measures applied to Sample 1 (e.g., IPDE, NEO PI-R and FFM-PD counts), the following instruments were used in the clinical sample:

Structured Clinical Interview for DSM-IV Axis I Disorders (SCID-I). SCID-I is a semi-structured interview used for making the major DSM-IV Axis I diagnoses (First, Spitzer, Gibbon, \& Williams, 1995). It evaluates affective, psychotic, substance-related, anxiety, somatoform, eating, and adaptive disorders.

Structured Clinical Interview for DSM-IV Axis II Personality Disorders (SCID-II). SCID-II is a semi-structured interview assessing DSM-IV Axis II PDs from the categorical approach (First, Gibbon, \& Spitzer, 1997). It is considered to be the gold standard semi-structured assessment instrument for PD.

\section{Procedure}

Validation Sample 1 was obtained in the Universitat Jaume I of Castelló from the last follow-up of two different prospective studies performed during $2009(n=327)$ and $2010(n=193)$ and one cross-sectional study from $2012(n=$ 222). All participants provided informed consent to participate in the study and received an amount of $€ 30$ for their collaboration.

Clinical Sample 2 was recruited from the outpatients' service of the Department of Mental Health 2 at the General Hospital of Castellón between 2005 and 2010. Healthcare professionals invited users to participate in the study. After signing their informed consent form, all subjects completed the IPDE. Patients were then evaluated in two sessions by a Clinical Psychologist or a Clinical Psychology Resident using the SCID-I and SCID-II. Between both assessment sessions, participants completed the NEO PI-R. Two hundred twenty were interviewed to confirm categorical diagnosis. $48 \%$ met categorical criteria for an Axis II disorder as determined by SCID-II, 91\% for at least one Axis I disorder as determined by SCID-I and 42\% met criteria for Axis I and Axis II disorder at the same time. The most common primary axis I diagnoses were affective $(37 \%)$, anxiety $(24 \%)$ and adaptive disorders $(19 \%)$. The most common Axis II diagnoses corre- sponded to cluster C (20\%), cluster B (14\%) and cluster A $(6 \%)$.

This study was approved by Universitat Jaume I and General Hospital of Castellón Ethics Committees.

\section{Statistical analysis}

First, we developed the normative data of the FFM-PD counts in the Spanish population from the basic statistics and intercorrelation matrix of normative sample facets. We then calculated PD subclinical case prevalence based on IPDE manual and FFM-PD counts criteria, analyzing the distribution and agreement of both estimates. We also searched for associations between the data obtained with IPDE and NEO PI-R by techniques of analysis of variance, calculating effect size. Lastly, we analyzed convergent and divergent FFM-PD counts validity to estimate the subclinical cases identified with IPDE, calculating effect size after the Fisher's z transformation of $r$.

\section{Results}

In order to interpret the meaning of PD estimates from NEO PI-R facets, we achieved $T$-scores for each subject in the different PDs, applying the normative data compiled for the Spanish population. Since there were no statistically significant differences between male and female means ( $d=$ .11), this work used the normative data of the total sample. The benchmarks of FFM-PD counts are indicated in Table 11 .

Table 1. Spanish normative data and benchmarks of FFM-PD counts

\begin{tabular}{lccc}
\hline & \multicolumn{2}{c}{$T$-score $(M=50, S D=10)$} \\
\cline { 2 - 4 } FFM-PD & 50 & 60 & 65 \\
\hline Paranoid & $102-103$ & $124-125$ & $135-136$ \\
Schizoid & $100-101$ & $121-122$ & $131-132$ \\
Schizotypal & $88-89$ & $103-104$ & 111 \\
Antisocial & $210-212$ & $238-240$ & $253-254$ \\
Borderline & $115-116$ & $137-138$ & $148-149$ \\
Histrionic & $204-206$ & $228-229$ & $240-241$ \\
Narcissistic & $157-158$ & $178-180$ & $189-191$ \\
Avoidant & $142-144$ & $164-165$ & $174-175$ \\
Dependent & $62-63$ & $81-82$ & $91-92$ \\
Obsessive-Compulsive & $240-242$ & $263-265$ & $275-276$ \\
\hline
\end{tabular}

The basic statistics of DSM-PD estimation and FFM-PD counts are summarized in Table 2 and 3. The DSM-PD estimation with the IPDE questionnaire indicated in nine DSM-PD a greater number of detected subclinical cases in Sample 2 than in Sample 1, with median effect sizes of moderate magnitude $(M d n=.69)$ except Narcissistic PD $(d=$ .11). Mean T-scores in the FFM-PD counts of cluster $A$ and C for Sample 2 we higher than in Sample 1, with median effect sizes of large magnitude $(M d n=1.03$ and 1.12). Patients

1 Calculator for scoring the FFM-PD counts and normative data sheet for Spanish population are available from the first author. 
of Sample 2 diagnosed in any of the DSM-PDs according to SCID-II and compared to the negative diagnosis group, presented a greater number of detected PDs $(d=.72$ and .93 in DSM-PD and FFM-PD) and higher raw scores in estimated clusters, with median effect sizes of medium magnitude ( $M d n$ $=.66$ and .76 , respectively). The internal consistency coefficients of subclinical cases detected with IPDE $(M d n=.53)$ presented low reliability values ranging from .39 (Schizoid PD) to .68 (Avoidant PD) in Sample 1 and from .43 (Narcissistic PD) to .67 (Avoidant PD) in Sample 2. Internal FFMPD consistency coefficients $(M d n=.71)$ varied between .52 (Schizotypal PD) and .83 (Dependent PD) in Sample 1 and between .52 (Schizotypal PD) and .84 (OCPD) in Sample 2, indicating adequate reliability.

Table 2. Summary of descriptive statistics, correlation and internal consistency for DSM-PD

\begin{tabular}{lccccccccccccc}
\hline PD & 1 & 2 & 3 & 4 & 5 & 6 & 7 & 8 & 9 & 10 & $M$ & $S D$ & $\alpha_{1}$ \\
\hline 1. Paranoid & 1 & $.15^{*}$ & $.55^{* * *}$ & $.32^{* * *}$ & $.52^{* * *}$ & $.17^{*}$ & $.18^{* * *}$ & $.39^{* * *}$ & $.27^{* * *}$ & $.23^{* * *}$ & 2.53 & 1.72 & .58 \\
2. Schizoid & $.26^{* * *}$ & 1 & $.46^{* * *}$ & .11 & $.21^{* * *}$ & $-.24^{* * *}$ & $-.21^{* * *}$ & $.37^{* * *}$ & .03 & .04 & 2.88 & 1.64 & .49 \\
3. Schizotypal & $.53^{* * *}$ & $.49^{* * *}$ & 1 & $.35^{* * *}$ & $.55^{* * *}$ & .05 & .07 & $.50^{* * *}$ & $.29^{* * *}$ & $.28^{* * *}$ & 2.71 & 1.97 & .60 \\
4. Antisocial & $.34^{* * *}$ & $.19^{* * *}$ & $.34^{* * *}$ & 1 & $.39^{* * *}$ & $.26^{* * *}$ & $.22^{* * *}$ & $.18^{* * *}$ & $.17^{*}$ & .09 & 1.00 & 1.23 & .52 \\
5. Borderline & $.45^{* * *}$ & $.20^{* * *}$ & $.45^{* * *}$ & $.35^{* * *}$ & 1 & $.37^{* * *}$ & $.22^{* * *}$ & $.46^{* * *}$ & $.48^{* * *}$ & $.33^{* * *}$ & 4.31 & 2.10 & .63 \\
6. Histrionic & $.20^{* * *}$ & -.07 & $.17^{* * *}$ & $.27^{* * *}$ & $.43^{* * *}$ & 1 & $.34^{* * *}$ & -.01 & $.34^{* * *}$ & $.19^{* * *}$ & 3.10 & 1.71 & .49 \\
7. Narcissistic & $.38^{* * *}$ & .06 & $.30^{* * *}$ & $.43^{* * *}$ & $.32^{* * *}$ & $.39^{* * *}$ & 1 & -.04 & .01 & $.27^{* * *}$ & 2.16 & 1.51 & .43 \\
8. Avoidant & $.46^{* * *}$ & $.34^{* * *}$ & $.51^{* * *}$ & $.12^{* * *}$ & $.45^{* * *}$ & .07 & $.15^{* * *}$ & 1 & $.42^{* * *}$ & $.37^{* * *}$ & 4.17 & 2.17 & .67 \\
9. Dependent & $.33^{* * *}$ & $.08^{*}$ & $.32^{* * *}$ & $.22^{* * *}$ & $.47^{* * *}$ & $.36^{* * *}$ & $.16^{* * *}$ & $.40^{* * *}$ & 1 & $.26^{* * *}$ & 2.96 & 1.97 & .65 \\
10. Obsessive & $.38^{* * *}$ & $.18^{* * *}$ & $.36^{* * *}$ & $.17^{* * *}$ & $.34^{* * *}$ & $.15^{* * *}$ & $.29^{* * *}$ & $.42^{* * *}$ & $.28^{* * *}$ & 1 & 3.55 & 1.75 & .48 \\
$M$ & 1.57 & 1.42 & 1.19 & 0.70 & 2.05 & 2.57 & 2.34 & 2.75 & 1.72 & 2.76 & & & \\
SD & 1.35 & 1.17 & 1.47 & 0.99 & 1.78 & 1.67 & 1.65 & 2.03 & 1.48 & 1.62 \\
$\alpha_{2}$ & .51 & .39 & .59 & .43 & .60 & .46 & .59 & .68 & .54 & .47 &
\end{tabular}

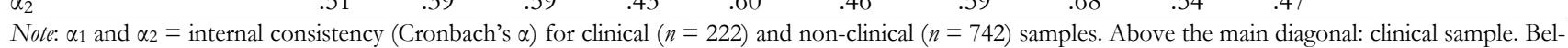
low the main diagonal: non-clinical sample.

${ }^{*} p<.05^{* * *} p<.001$ (two-tailed).

Table 3. Summary of descriptive statistics, correlation and internal consistency for FFM-PD.

\begin{tabular}{|c|c|c|c|c|c|c|c|c|c|c|c|c|c|}
\hline PD & 1 & 2 & 3 & 4 & 5 & 6 & 7 & 8 & 9 & 10 & $M$ & $S D$ & $\alpha_{1}$ \\
\hline 1. Paranoid & 1 & $.47^{* * *}$ & $.57^{* * *}$ & $.58^{* * *}$ & $.50^{* * *}$ & $-.36^{* * * *}$ & $.74^{* * * *}$ & $.32^{* * * *}$ & $.40^{* * *}$ & -.10 & 134.63 & 27.73 & .78 \\
\hline 2. Schizoid & $.53^{* * *}$ & 1 & $.74^{* * *}$ & $-.31^{* * *}$ & .07 & $-.87^{* * * *}$ & -.09 & $.84^{* * *}$ & $.48^{* * *}$ & $.14^{*}$ & 130.48 & 25.80 & .77 \\
\hline 3. Schizotypal & $.57^{* * * *}$ & $.66^{* * *}$ & 1 & -.03 & $.48^{* * *}$ & $-.56^{* * *}$ & .10 & $.76^{* * * *}$ & $.74^{* * *}$ & $-.22^{* * *}$ & 117.02 & 20.20 & .52 \\
\hline 4. Antisocial & $.48^{* * *}$ & $-.33^{* * *}$ & .01 & 1 & $.43^{* * *}$ & $.45^{* * *}$ & $.90^{* * *}$ & $-.47^{* * *}$ & .03 & $-.53^{* * *}$ & 222.43 & 34.96 & .71 \\
\hline 5. Borderline & $.33^{* * *}$ & -.04 & $.48^{* * *}$ & $.36^{* * *}$ & 1 & .13 & $.36^{* * * *}$ & $.30^{* * * *}$ & $.74^{* * *}$ & $-.34^{* * *}$ & 157.80 & 24.75 & .70 \\
\hline 6. Histrionic & $-.47^{* * *}$ & $-.86^{* * *}$ & $-.45^{* * *}$ & $.44^{* * *}$ & $.25^{* * *}$ & 1 & $.14^{*}$ & $-.77^{* * *}$ & $-.25^{* * *}$ & $-.45^{* * *}$ & 191.87 & 26.23 & .58 \\
\hline 7. Narcissistic & $.72^{* * *}$ & -.04 & $.15^{* * *}$ & $.86^{* * *}$ & $.23^{* * *}$ & .04 & 1 & $-.31^{* * * *}$ & .01 & $-.25^{* * *}$ & 164.52 & 27.97 & .66 \\
\hline 8. Avoidant & $.36^{* * * *}$ & $.82^{* * *}$ & $.70^{* * *}$ & $-.51^{* * *}$ & $.26^{* * * *}$ & $-.72^{* * *}$ & $-.31^{* * *}$ & 1 & $.71^{* * * *}$ & $.17^{*}$ & 183.96 & 28.64 & .72 \\
\hline 9. Dependent & $.35^{* * *}$ & $.40^{* * *}$ & $.68^{* * *}$ & .00 & $.78^{* * *}$ & $-.15^{* * *}$ & -.03 & $.68^{* * *}$ & 1 & $-.35^{* * *}$ & 136.78 & 19.88 & .82 \\
\hline 10. Obsessive & .02 & $.26^{* * *}$ & $-.21^{* * *}$ & $-.58^{* * *}$ & $-.38^{* * *}$ & $-.60^{* * * *}$ & $-.22^{* * *}$ & $.28^{* * *}$ & $-.27^{* * *}$ & 1 & 225.55 & 27.66 & .84 \\
\hline M & 122.16 & 102.01 & 98.61 & 243.74 & 142.13 & 217.21 & 174.43 & 148.18 & 84.52 & 210.59 & & & \\
\hline$S D$ & 23.58 & 21.57 & 17.04 & 29.78 & 22.27 & 24.97 & 23.41 & 23.86 & 21.80 & 26.04 & & & \\
\hline$\alpha_{2}$ & .78 & .79 & .52 & .69 & .73 & .68 & .64 & .71 & .83 & .70 & & & \\
\hline
\end{tabular}

Note: $\alpha_{1}$ and $\alpha_{2}=$ internal consistency (Cronbach's $\left.\alpha\right)$ for clinical $(n=222)$ and non-clinical $(n=742)$ samples. Above the main diagonal: clinical sample. Bellow the main diagonal: non-clinical sample.

${ }^{*} p<.05{ }^{* * *} p<.001$ (two-tailed)

Table 4 summarizes prevalence data of subclinical cases and the consistency of results obtained with the estimates of PD symptoms and FFM-PD. In Sample 1, a total of 654 subjects with IPDE scores compatible with subclinical case estimations $(88 \%)$ were detected, $78 \%$ showing more than one PD $(M=3.00, S D=2.31)$. With the FFM-PD approach, 517 people $(70 \%)$ reached $T \geq 65$, presenting a $75 \%$ estimate of over one PD $(M=1.94, S D=1.79)$. The percentage of agreement between estimates made with IPDE and FFM-PD counts was high in cluster A of the DSM-IV (81\%), medium in cluster B $(68 \%)$ and low in cluster $\mathrm{C}(63 \%)$, agreeing in $72 \%$ of estimates for any PD. In Sample 2, on the other hand, 216 subjects were detected with IPDE scores compatible with subclinical case estimations (97\%), with some 95\% showing more than one PD $(M=5.41, S D=2.24)$. With the FFM-PD approach, 202 people $(90 \%)$ were considered subclinical cases $(T \geq 65), 85 \%$ presenting an estimate of more than one $\mathrm{PD}(M=3.94, S D=2.07)$. The percentage of agreement between estimates made with IPDE and FFM-PD counts was medium in cluster A (68\%) and cluster B (67\%), and low in cluster C (56\%), reaching $92 \%$ agreement in the estimation of any PD. We must highlight the lack of agreement between subclinical cases of OCPD (48\%) in Sample 1, and OCPD (30\%) and Histrionic PD (42\%) in Sample 2. Furthermore, the value of sensitivity for any PD indicated that FFM-PD counts correctly identified $73 \%$ and $93 \%$ of subclinical cases detected by IPDE in Sample 1 and 2, respectively. The high score in the positive predictive power of 
any PD (PPP $=93 \%$ and $99 \%$ in Sample 1 and 2 ) suggested a high probability of exceeding $T \geq 65$ in FFM-PD threshold when IPDE indicates the presence of a subclinical case of PD. The odds ratio values suggested positive association between PDs estimates with IPDE and FFM-PD counts in both samples, with the exceptions being OCPD in Sample 1 and OCPD and Histrionic PD in Sample 2. The estimate of any FFM-PD in Sample 1 and 2 was 3.80 and 25 times more likely in a subclinical case detected with IPDE than in an unidentified subject as subclinical case with this questionnaire.

Table 4. Diagnostic agreement between DSM-PD symptom counts and FFM-PD counts.

\begin{tabular}{|c|c|c|c|c|c|c|c|c|c|c|c|c|c|c|c|c|}
\hline \multirow[b]{3}{*}{ Paranoid } & \multicolumn{2}{|c|}{ DSMPD } & \multicolumn{2}{|c|}{ FFMPD } & \multicolumn{2}{|c|}{ SEN } & \multicolumn{2}{|c|}{ SPE } & \multicolumn{2}{|c|}{ PPP } & \multicolumn{2}{|c|}{ NPP } & \multicolumn{2}{|c|}{ OR } & \multicolumn{2}{|c|}{$95 \% \mathrm{CI}$} \\
\hline & $\mathrm{S} 1$ & $\mathrm{~S} 2$ & $\mathrm{~S} 1$ & $\mathrm{~S} 2$ & S1 & S2 & S1 & $\mathrm{S} 2$ & S1 & $\mathrm{S} 2$ & S1 & $\mathrm{S} 2$ & S1 & $\mathrm{S} 2$ & $\mathrm{~S} 1$ & $\mathrm{~S} 2$ \\
\hline & 22 & 48 & 25 & 45 & .55 & .64 & .84 & .72 & .49 & .67 & .87 & .69 & 6.28 & 4.50 & $4.22-9.34$ & $2.46-8.27$ \\
\hline Schizoid & 16 & 58 & 9 & 46 & .27 & .63 & .95 & .77 & .51 & .79 & .87 & .61 & 7.45 & 5.64 & $4.24-13.12$ & $2.98-11.74$ \\
\hline Schizotypal & 17 & 50 & 20 & 58 & .52 & .75 & .87 & .58 & .44 & .64 & .90 & .70 & 6.94 & 4.05 & $4.46-10.80$ & $2.21-7.47$ \\
\hline Antisocial & 6 & 13 & 38 & 17 & .86 & .61 & .65 & .89 & .13 & .45 & .99 & .94 & 11.14 & 12.73 & $4.42-29.86$ & 4.85-33.99 \\
\hline Borderline & 33 & 60 & 37 & 3 & .70 & .75 & .79 & .71 & .62 & .90 & .84 & .44 & 8.57 & 7.33 & $5.96-12.33$ & $3.43-15.87$ \\
\hline Histrionic & 47 & 78 & 16 & 64 & .22 & .04 & .89 & .01 & .65 & .01 & .57 & .41 & 2.44 & & $1.59-3.74$ & \\
\hline Narcissistic & 36 & 33 & 24 & 15 & .45 & .24 & .88 & .90 & .67 & .55 & .74 & .70 & 5.82 & 2.85 & $3.99-8.52$ & $1.26-6.46$ \\
\hline Avoidant & 48 & 76 & 11 & 64 & .22 & .74 & .98 & .67 & .93 & .87 & .58 & .46 & 18.18 & 5.81 & $7.50-46.94$ & 2.85-11.94 \\
\hline Dependent & 24 & 55 & 13 & 77 & .33 & .89 & .93 & .36 & .59 & .63 & .81 & .72 & 6.26 & 4.34 & $3.92-10.02$ & $2.07-9.19$ \\
\hline Obsessive & 52 & 71 & 0 & 3 & .01 & .03 & 1 & .97 & .67 & .71 & .48 & .29 & 1.82 & 1.01 & $0.13-50.86$ & $.17-7.76$ \\
\hline Any PD & 88 & 97 & 70 & 91 & .73 & .93 & .58 & .67 & .93 & .99 & .23 & .20 & 3.80 & 25.00 & $2.35-6.16$ & $3.53-216.20$ \\
\hline
\end{tabular}

Note: DSMPD $=$ percent of subclinical cases detected with IPDE; FFMPD = percent of estimates with FFM-PD counts; SEN = sensitivity of FFMPD on $\mathrm{IPDE}$ detection; $\mathrm{SPE}=$ specificity; $\mathrm{PPP}=$ positive predictive power; $\mathrm{NPP}=$ negative predictive power; $\mathrm{OR}=$ odds ratio; $\mathrm{CI}=$ confidence interval. $\mathrm{S} 1=$ non-clinical sample $(n=742) ; \mathrm{S} 2=$ clinical sample $(n=222)$.

Moreover, the IPDE scores of individuals exceeding the FFM-PD threshold $(T \geq 65)$ were compared with those scoring below this cutoff point to verify the association between DSM-PD and FFM-PD estimates (Table 5). The variance analysis showed in both samples significant $F$ values in all PDs, except in OCPD. The FFM-PD counts differed signifi- cantly depending on whether subjects classified as a subclinical case in the DSM-PD, rejecting the null hypothesis of means equality. Effect sizes $\left(\eta^{2}\right)$ were large in most PDs $(M d n=.12$ in Sample 1 and 2), except small magnitude for Histrionic PD and OCPD in Sample 1 and 2, and Narcissist PD in Sample 2.

Table 5. Between groups mean differences for DSM-PD symptom counts based on FFM-PD counts.

\begin{tabular}{|c|c|c|c|c|c|c|c|c|}
\hline \multirow[b]{3}{*}{ FFM-PD } & \multicolumn{8}{|c|}{ DSM-PD } \\
\hline & \multicolumn{2}{|c|}{$n=742$} & \multirow[b]{2}{*}{$F$} & \multirow[b]{2}{*}{$\eta^{2}$} & \multicolumn{2}{|c|}{$n=222$} & \multirow[b]{2}{*}{$F$} & \multirow[b]{2}{*}{$\eta^{2}$} \\
\hline & $T<65$ & $T \geq 65$ & & & $T<65$ & $T \geq 65$ & & \\
\hline Paranoid & $1.24(557)$ & $2.54(185)$ & $118.40^{* * *}$ & .14 & $1.84(121)$ & 3.35 (101) & $32.34^{* * *}$ & .13 \\
\hline Schizoid & $1.28(557)$ & $2.79(67)$ & $75.68^{* * *}$ & .09 & $2.26(119)$ & $3.60(103)$ & $40.69^{* * *}$ & .16 \\
\hline Schizotypal & $0.85(595)$ & $2.56(147)$ & $110.58^{* * *}$ & .13 & 1.77 (93) & 3.39 (129) & $26.92^{* * *}$ & .11 \\
\hline Antisocial & $0.39(461)$ & $1.22(281)$ & $45.87^{* * *}$ & .06 & 0.77 (184) & $2.11(38)$ & $52.74^{* * *}$ & .19 \\
\hline Borderline & $1.36(468)$ & $3.23(274)$ & $211.74^{* * *}$ & .22 & $2.82(79)$ & $5.13(143)$ & $41.51^{* * *}$ & .16 \\
\hline Histrionic & $2.40(622)$ & $3.48(120)$ & $19.57^{* * * *}$ & .03 & $3.02(216)$ & $6.17(6)$ & $4.09^{*}$ & .02 \\
\hline Narcissistic & $1.91(561)$ & 3.65 (181) & $115.95^{* * *}$ & .14 & 1.98 (189) & $3.15(33)$ & $8.06^{* *}$ & .04 \\
\hline Avoidant & $2.43(657)$ & $5.15(85)$ & $87.23^{* * *}$ & .10 & $2.96(79)$ & $4.84(143)$ & $34.47^{* * *}$ & .14 \\
\hline Dependent & $1.25(481)$ & $2.59(261)$ & $143.18^{* * *}$ & .16 & $1.60(50)$ & 3.35 (172) & $20.52^{* * *}$ & .08 \\
\hline Obsessive & $2.75(739)$ & $4.33(3)$ & 0.24 & .00 & $3.53(215)$ & $4.00(7)$ & 0.00 & .00 \\
\hline
\end{tabular}

Note: $T=$ standardized score $(M=50, S D=10) . M(n)$.

Cohen's operational definitions for small, medium and large effect sizes are $\eta^{2}=.01, .06$ and .14 , respectively.

${ }^{*} p<.05^{* *} p<.01^{* * *} p<.001$

Finally, we analyzed construct validity and calculated coefficients for convergent and discriminant validity (Table 6). On the one hand, the FFM-PD counts in Sample 1 presented a median convergent correlation of $r=.48$ with their equivalent subclinical cases of IPDE, within a range of .24 (OCPD) and .58 (Borderline PD). Applying attenuation correction, the average convergent correlation reached a value of $r=.74$, with a range of .42 (OCPD) to .94 (Schizotypal $\mathrm{PD})$. Convergent correlations were statistically significant and outperformed any divergent correlation with effect size magnitude at least small $(q>.10)$, except Histrionic -
Avoidant PDs. Convergent correlations also exceeded average divergent correlations in all FFM-PD with an effect size of medium magnitude $(q \geq .30)$ or at least small magnitude (Paranoid and Dependent PDs, with $q \geq .20$ ). However, we detected a divergent validity coefficient (Paranoid, Schizoid, Schizotypal and Dependent PDs) and two divergent validity coefficients (OCPD and Histrionic PD) of greater magnitude than the equivalent convergent validity coefficients. No significant size effect was reached except in the aforementioned case (Histrionic - Avoidant PDs). On the other hand, the FFM-PD counts in Sample 2 showed a median convergent 
correlation of .50 with their equivalent subclinical cases of IPDE, within a range of .15 (OCPD) and .65 (Borderline PD). Applying attenuation correction, the average convergent correlations reached a value of .76, with an amplitude of .24 (OCPD) to .98 (Schizotypal PD). Convergent correlations were statistically significant and exceeded any divergent correlation according to the effect size magnitude which was at least small $(q>.10)$, with one exception (OCPD with Antisocial, Borderline and Histrionic PDs). Convergent correla- tions also outperformed average divergent correlations in all FFM-PD with an effect size of medium magnitude (Schizoid, Histrionic and Avoidant PDs, where $q \geq .30$ ) or at least small magnitude (except Paranoid PD and OCPD). However, at a singular level a divergent validity coefficient (Paranoid, Schizotypal and Narcissistic PDs) and three divergent validity coefficients (OCPD) of greater magnitude than the equivalent convergent validity coefficients were detected, without reaching significant size effect except for OCPD.

Table 6. Construct validity of FFM-PD counts with DSM-PD symptom counts.

\begin{tabular}{|c|c|c|c|c|c|c|c|c|}
\hline \multirow[b]{3}{*}{ FFM-PD } & \multicolumn{8}{|c|}{ DSM-PD } \\
\hline & \multicolumn{4}{|c|}{$n=742$} & \multicolumn{4}{|c|}{$n=222$} \\
\hline & $\mathrm{CV}$ & MDV & $q$ & $\mathrm{DV}>\mathrm{CV}$ & $\mathrm{CV}$ & MDV & $q$ & $\mathrm{DV}>\mathrm{CV}$ \\
\hline Paranoid & $.49^{* * *}$ & .32 & .20 & 1 & $.49^{* * *}$ & .29 & .17 & 1 \\
\hline Schizoid & $.42^{* * *}$ & .12 & .33 & 1 & $.55^{* * *}$ & .08 & .42 & 0 \\
\hline Schizotypal & $.52^{* * *}$ & .27 & .31 & 1 & $.55^{* * *}$ & .24 & .26 & 1 \\
\hline Antisocial & $.44^{* * *}$ & .17 & .32 & 1 & $.46^{* * *}$ & .17 & .26 & 0 \\
\hline Borderline & $.58^{* * *}$ & .26 & .40 & 0 & $.65^{* * *}$ & .31 & .27 & 0 \\
\hline Histrionic & $.29^{* * *}$ & -.15 & .45 & 2 & $.46^{* * *}$ & -.09 & .34 & 0 \\
\hline Narcissistic & $.50^{* * *}$ & .21 & .34 & 0 & $.38^{* * *}$ & .17 & .20 & 1 \\
\hline Avoidant & $.55^{* * *}$ & .12 & .50 & 0 & $.51^{* * *}$ & .11 & .36 & 0 \\
\hline Dependent & $.47^{* * *}$ & .27 & .23 & 1 & $.55^{* * *}$ & .25 & .25 & 0 \\
\hline Obsessive & $.24^{* *}$ & -.11 & .36 & 2 & -.16 & -.13 & .04 & 3 \\
\hline
\end{tabular}

Note: $\mathrm{CV}=$ convergent validity; $\mathrm{MDV}=$ mean of divergent validities; $q=$ effect sizes of differences between $\mathrm{CV}$ and $\mathrm{MDV} ; \mathrm{DV}>\mathrm{CV}=$ number of divergent coefficients higher than convergent correlations.

Cohen's operational definitions for small, medium and large effect sizes of correlation differences $\left(\mathrm{z}_{1}-z_{2}\right)$ are $q=.10, .30$ and .50 , respectively. Criterion: $q>$ 10 .

${ }^{* *} p<.01 \quad{ }^{* * *} p<.001$ (two-tailed)

\section{Discussion and conclusions}

There is widespread interest in using dimensional models of personality to analyze and describe personality pathology (American Psychiatric Association, 2013), FFM which shows greater consensus and empirical support (Widiger \& Costa, 2013). Empirical evidence indicates that the best way to describe, evaluate and diagnose PDs is a model conceiving them as a continuum with normal personality and considering the differences between normal and maladaptive personality as gradual and quantitative rather than qualitatively different systems (Samuel \& Widiger, 2008; Saulsman \& Page, 2004; Widiger \& Trull, 2007). In fact, the FFM provides a reasonably complete integration of normal and abnormal personality, explaining the abnormal personality functioning within the same model and language used to describe the general structure of personality (Gore \& Widiger, 2013; Mullins-Sweatt \& Widiger, 2006; Trull \& Widiger, 2013). On the other hand, the derived methodological development to assess PDs from NEO PI-R facets is also relevant, with simple techniques applied in daily practice and normative data to interpret results (Miller, 2012). Both contributions, together with the evaluation of personal functioning in social and workplace fields, are useful in carrying out PD diagnosis. Following this research line, we firstly developed Spanish normative data to describe and interpret FFM-PD counts. Secondly, their preliminary validation was presented in two
Spanish samples in order to test FFM-PD count approach utility in describing and analyzing DSM-PD.

In order to assess PDs from a dimensional perspective, in which they are conceived as extreme variants of general traits in the continuum of normal and abnormal personality (Gore \& Widiger, 2013; Trull \& Widiger, 2013; Widiger \& Costa, 2012), the representative values of scores obtained in the Spanish population with FFM-PD count method was calculated. The statistical deviation of each PD referred to in the DSM-IV was considered. The resulting normative data were derived from studies done during the Spanish adaptation of the NEO PI-R (Costa \& McCrae, 2008) and from works defining PD prototypes (Lynam \& Widiger, 2001; Miller \& Lynam, 2008). This contribution has the value of providing benchmarks of FFM-PD counts for estimating personality pathology in the Spanish population, without using specific gender norms in the light of our data and findings from previous studies (Miller et al., 2008; Samuel et al., 2010; Samuel \& Widiger, 2009).

Although it could be accepted that PDs are among the most common mental disorders in the general population (American Psychiatric Association, 2013), subclinical case estimations reached high levels in our samples: $88 \%$ with the IPDE screening questionnaire and $70 \%$ with FFM-PD counts in Sample 1, and $97 \%$ and $91 \%$, respectively, in Sample 2. PD screening with the IPDE questionnaire tends to produce a considerable number of false positives (LópezIbor et al., 1996). 
Percent agreement and consistency between IPDE and FFM-PD estimates in our work and the diagnostic capability of FFM-PD values suggest FFM-PD counts validity to identify people not achieving any subclinical IPDE threshold. This confirms that FFM-PD counts offers some benefits of screening questionnaires and reduces the number of detected subclinical cases and PD amounts. On the one hand, with the exception of OCPD and Histrionic PD, FFM-PD counts are relatively successful in capturing constructs estimated with an explicit DSM-PD measure in both samples. On the other hand, according to the Receiver Operating Characteristics (ROC) analyses in Sample 2, benchmarks in three FFMPDs (Histrionic, Narcissistic, OCPD) would need a significant reduction to maintain diagnostic efficiency (SEN > .80).

In addition to a high degree of PD overlap and a significant number of shared facets in FFM-PD counts, the convergent validity of this technique has been demonstrated in overcoming divergent correlation coefficients between FFMPD counts and DSM-PD symptom counts estimation, reaching a median $r=.48$ in Sample 1 and .50 in Sample 2. These results are similar to those in other normative studies (Miller et al., 2008), although validity coefficients could be attenuated by inadequate IPDE questionnaire reliability measurement values in both our samples. As for the mean of discriminant validity coefficients, it had an absolute median correlation value of .19 in Sample 1 and .17 in Sample 2. Overall, there were eight PDs in Sample 1 and two PDs in Sample 2 with medium effect sizes of differences between convergent and discriminant coefficients and two and six PDs, respectively, with small effect sizes. Our results support the construct validity of seven FFM-PD counts, with weakest support for OCPD, Paranoid, and Histrionic PDs, which should be used with caution. In the current work such validity was not contrasted with the calibration reference, since the IPDE diagnostic interview was not conducted in any sample and the dimensional data of SCID-II in Sample 2 were impossible to use.

Normative data for FFM-PD counts may be valid for basic research and applied psychology with Spanish samples, providing benchmarks so far non-existent for PD interpretation and description. Theoretically, these normative data can further clarify PD relationships and differences and provide a more accurate interpretation of their meaning from the FFM perspective, as the facets in each PD prototype would be known and normative Spanish population data would be available. The difficulties in OCPD and Histrionic PD, with lowest agreement degree between the two estimates, diagnostic parameters and convergent correlation, confirmed FFM-PD inability to estimate these PDs. In the case of OCPD, this relates to NEO PI-R inefficiency to evaluate the maladaptive personality in the pole of high responsibility, as $90 \%$ of items in this dimension are related to the adaptive personality evaluation (Bastiaansen, Rossi, \& De Fruyt, 2013; Miller et al., 2008; Samuel \& Gore, 2012; Samuel \& Widiger, 2011). Similar difficulties concur in Histrionic PD, with this PD associated to high scores on personality traits which may also be inadequately assessed in NEO PI-R. This is so because few elements of this questionnaire are designed to evaluate maladaptivity at the upper ends of relevant facets from the Extraversion, Openness and Agreeableness dimensions (Bastiaansen et al., 2013; Miller et al., 2008; Samuel \& Gore, 2012).

FFM-PD counts from the measurement of normal personality traits, in addition to using a model where all PD incorporate facets of different dimensions of personality, can facilitate the clinical or behavioral evaluation process with a detection technique providing accurate descriptions of the basic personality facets and suitable for integration within an explicit clinical disorder assessment proposal from the FFM perspective (Widiger \& Presnall, 2013). It also allows psychologists to compare FFM-PD counts with normative data valid to rigorously assess the presence of personality pathology and, where appropriate, specify therapeutic targets. Content information is understandable for communicating results at personal or expert level, avoiding possible diagnostic label stigmatization and legal complications sometimes associated to the application of psychopathological questionnaires. Its utility is proven in clinical and organizational fields, facilitating the psychological diagnosis process (Miller et al., 2010; Widiger \& Mullins-Sweatt, 2010; Widiger \& Presnall, 2013) and counterproductive behavior identification or competency assessment in the workplace (De Fruyt et al., 2009; Wille, De Fruyt, \& De Clercq, 2013). This approach also helps to overcome some categorical model difficulties, such as PD comorbidity (Clark, 2007; Trull \& Durrett, 2005; Widiger \& Samuel, 2005). In our study, the cooccurrence of DSM-PD categories was evident in the number of estimated PDs per participant especially with IPDE screening $(M=3.0$ and 5.41 in Sample 1 and Sample 2, respectively).

In the present work PDs were measured with self-report methodology as a first step toward diagnosis (Samuel, 2015). The questionnaires, which involve cost and time reductions and provide important psychometric rigor, can limit the result accuracy by social desirability, distortion, emotional state or response trends when used exclusively (Huprich, Bornstein, \& Schmitt, 2011), as it happens with many PD-related studies. This methodology was also used for personality assessment, as its practice with self-reports remains prevalent over other methods in various contexts. The NEO PI-R has exceptional psychometric properties and can be easily applied, although it is affected by the social desirability effect due to the standardization sample characteristics. In addition to these common methodological limitations, some specifics in our work relate to sampling and exploratory nature. We used convenience samples with a recruitment system not allowing generalization of IPDE questionnaire comparison results, serving exclusively for development of Spanish normative data and initial FFM-PD count technique validation. Therefore, our future research should validate the FFM-PD count method for screening personality pathology with random or stratified clinical samples and dimensional screening 
instruments attached to a subsequent diagnostic interview. If dimensional scores in the SCID-II were available, these would have been expected to increase the convergent validity of our measures as continuous psychopathology measures are preferable to categorical measures and have demonstrated considerable advantages for validity (Nestadt et al., 2012; Samuel, 2015).

In sum, an approximation is made to a line of innovative research on personality pathology linked to a shift in the PD categorical paradigm and FFM-PD count approach. It is possible to benefit from a dimensional PD detection method, based on the scientific evidence resulting from the dominant personality structure model, the theoretical basis of experts in personality psychopathology research, and the practical criterion provided by psychological PD treatment experts. We now have specific normative data and a standard-

\section{References}

American Psychiatric Association. (2000). Diagnostic and Statistical Manual of Mental Disorders-Text Revision (DSM-IV-TR). Washington, DC: Author.

American Psychiatric Association. (2013). Diagnostic and Statistical Manual of Mental Disorders (5th ed.). Arlington, VA: Author.

Bagby, R. M., Costa, P. T., Widiger, T. A., Ryder, A. G., \& Marshall, M. (2005). DSM-IV personality disorders and the five-factor model of personality: A multi-method examination of domain-and facet-level predictions. European Journal of Personality, 19(4), 307-324. doi: $10.1002 /$ per. 563

Bastiaansen, L., Rossi, G., \& De Fruyt, F. (2013). Comparing five sets of five-factor model personality disorder counts in a heterogeneous sample of psychiatric patients. European Journal of Personality, 27(4), 377-388. doi:10.1002/per.1859

Clark, L. A. (2007). Assessment and diagnosis of personality disorder: Perennial issues and an emerging reconceptualization. Annual Review of Psychology, 58, 227-257. doi:10.1146/annurev.psych.57.102904.190200

Costa, P. T., \& McCrae, R. R. (2008). Inventario de Personalidad NEO Revisado (NEO PI-R). Inventario NEO Reducido de Cinco Factores (NEO-FFI). Manual Revised NEO personality inventory (NEO PI-R) and NEO Five-Factor inventory (NEO FFI). Manual] (3rd ed.). Madrid, Spain: TEA Ediciones.

De Fruyt, F., De Clercq, B. J., Miller, J., Rolland, J., Jung, S., Taris, R., ... Van Hiel, A. (2009). Assessing personality at risk in personnel selection and development. European Journal of Personality, 23(1), 51-69. doi:10.1002/per.703

First, M. B., Gibbon, M., \& Spitzer, R. L. (1997). User's guide for the structured clinical interview for DSM-IV axis II personality disorders: SCID-II. Washington, DC: American Psychiatric Pub.

First, M. B., Spitzer, R. L., Gibbon, M., \& Williams, J. B. (1995). Structured clinical interview for DSM-IV axis I disorders. New York, NY: New York State Psychiatric Institute.

Gore, W. L., \& Widiger, T. A. (2013). The DSM-5 dimensional trait model and five-factor models of general personality. Journal of Abnormal Psychology, 122(3), 816-821. doi:10.1037/a0032822

Hengartner, M. P., Müller, M., Rodgers, S., Rössler, W., \& Ajdacic-Gross, V. (2014). Interpersonal functioning deficits in association with DSMIV personality disorder dimensions. Social Psychiatry and Psychiatric Epidemiology, 49(2), 317-325. doi:10.1007/s00127-013-0707-x

Huprich, S. K., Bornstein, R. F., \& Schmitt, T. A. (2011). Self-report methodology is insufficient for improving the assessment and classification of Axis II personality disorders. Journal of Personality Disorders, 25(5), 557-570. doi:10.1521/pedi.2011.25.5.557

John, O. P., Naumann, L. P., \& Soto, C. J. (2008). Paradigm shift to the integrative Big Five trait taxonomy: History, measurement, and conceptual issues. In O. P. John, R. W. Robins, \& L. A. Pervin (Eds.), Hand- ized profile facilitating the interpretation of the results of a method to estimate the PDs in Spanish studies from the FFM perspective in different psychological areas. The FFMPD count technique serves not only to reproduce the DSMPD classification system and estimate other personality dysfunctions, but to potentially contribute to improving the clinical description and diagnosis of PDs considered as maladaptive variants of normal personality.

Acknowledgements: This work was supported by the Autonomous Government of Valencia, Spain (Grant GV/2016/158). LCC was supported by a post-doctoral fellowship from the Fundación Séneca (Seneca Foundation, Regional Agency for Science and Technology, Murcia, Spain, 19151/PD/13) and a QIMR Berghofer Fellowship, Australia. We thank Donald R. Lynam and Joshua D. Miller for their suggestions in this work.

book of Personality: Theory and Research (3rd ed., pp. 114-158). New York, NY: Guilford Press.

López-Ibor, J., Pérez-Urdaniz, A., \& Rubio, V. (1996). Examen internacional de los trastornos de personalidad. Módulo DSM-IV [International Personality Disorder Examination. DSM-IV module]. Madrid, Spain: Meditor.

Loranger, A. W. (1995). International Personality Disorder Examination (IPDE). Geneva, Switzerland: World Health Organization.

Lynam, D. R. (2012). Assessment of maladaptive variants of five-factor model traits. Journal of Personality, 80(6), 1593-1613. doi:10.1111/j.14676494.2012.00775.x

Lynam, D. R., \& Widiger, T. A. (2001). Using the five-factor model to represent the DSM-IV personality disorders: An expert consensus approach. Journal of Abnormal Psychology, 110(3), 401-412. doi:10.1037/0021-843X.110.3.401

McCrae, R. R., \& Costa, P. T. (1997). Personality trait structure as a human universal. American Psychologist, 52(5), 509-516. doi:10.1037/0003066X.52.5.509

McCrae, R. R., Löckenhoff, C. E., \& Costa, P. T. (2005). A step toward DSM-V: Cataloguing personality-related problems in living. European Journal of Personality, 19(4), 269-286. doi:10.1002/per.564

Miller, J. D. (2012). Five-factor model personality disorder prototypes: A review of their development, validity, and comparison to alternative approaches. Journal of Personality, 80(6), 1565-1591. doi:10.1111/j.14676494.2012.00773.x

Miller, J. D., Bagby, R. M., Pilkonis, P. A., Reynolds, S. K., \& Lynam, D. R. (2005). A simplified technique for scoring DSM-IV personality disorders with the five-factor model. Assessment, 12(4), 404-415. doi:10.1177/1073191105280987

Miller, J. D., Few, L. R., Lynam, D. R., \& MacKillop, J. (2015). Pathological personality traits can capture DSM-IV personality disorder types. Per sonality Disorders: Theory, Research, and Treatment, 6(1), 32-40. doi:10.1037/per0000064

Miller, J. D., \& Lynam, D. R. (2008). Dependent personality disorder: comparing an expert generated and empirically derived five-factor model personality disorder count. Assessment, 15(1), 4-15. doi:10.1177/1073191107306095

Miller, J. D., Lynam, D. R., Rolland, J.-P., De Fruyt, F., Reynolds, S. K., Pham-Scottez, A., ... Bagby, R. M. (2008). Scoring the DSM-IV personality disorders using the five-factor model: Development and validation of normative scores for North American, French, and DutchFlemish samples. Journal of Personality Disorders, 22(5), 433-450. doi:10.1521/pedi.2008.22.5.433

Miller, J. D., Lynam, D. R., Widiger, T. A., \& Leukefeld, C. (2001). Personality disorders as extreme variants of common personality dimensions: 
Can the five-factor model adequately represent psychopathy? Journal of Personality, 69(2), 253-276. doi:10.1111/1467-6494.00144

Miller, J. D., Maples, J., Few, L. R., Morse, J. Q., Yaggi, K. E., \& Pilkonis, P. A. (2010). Using clinician-rated five-factor model data to score the DSM-IV personality disorders. Journal of Personality Assessment, 92(4), 296-305. doi:10.1080/00223891.2010.481984

Morey, L. C., Benson, K. T., Busch, A. J., \& Skodol, A. E. (2015). Personality disorders in DSM-5: Emerging research on the alternative model. Current Psycbiatry Reports, 17(4), 24. doi:10.1007/s11920-015-0558-0

Morey, L. C., Hopwood, C. J., Gunderson, J. G., Skodol, A. E., Shea, M. T., Yen, S., ... Sanislow, C. A. (2007). Comparison of alternative models for personality disorders. Psychological Medicine, 37(07), 983-994. doi:10.1017/S0033291706009482

Morey, L. C., \& Skodol, A. E. (2013). Convergence between DSM-IV-TR and DSM-5 diagnostic models for personality disorder: Evaluation of strategies for establishing diagnostic thresholds. Journal of Psychiatric Practice, 19(3), 179-193. doi:10.1097/01.pra.0000430502.78833.06

Mullins-Sweatt, S. N., \& Widiger, T. A. (2006). The Five-Factor model of personality disorder. A translation across science and practice. In R. F. Krueger \& J. L. Tackett (Eds.), Personality and Psychopathology (pp. 39-70). New York, NY: Guilford Press.

Mullins-Sweatt, S. N., \& Widiger, T. A. (2010). Personality-related problems in living: An empirical approach. Personality Disorders: Theory, Research, and Treatment, 1(4), 230-238. doi:10.1037/a0018228

Nestadt, G., Costa, P. T., Hsu, F.-C., Samuels, J., Bienvenu, O. J., \& Eaton, W. W. (2008). The relationship between the five-factor model and latent DSM-IV personality disorder dimensions. Comprebensive Psycbiatry, 49(1), 98-105. doi:10.1016/i.comppsych.2007.05.015

Nestadt, G., Di, C., Samuels, J., Cheng, Y.-J., Bienvenu, O., Reti, I., ... Bandeen-Roche, K. (2012). Concordance between personality disorder assessment methods. Psychological Medicine, 42(3), 657-667. doi:10.1017/S0033291711001632

O'Connor, B. P. (2005). Graphical analyses of personality disorders in fivefactor model space. European Journal of Personality, 19(4), 287-305. doi: $10.1002 /$ per. 558

Samuel, D. B. (2015). A review of the agreement between clinicians' personality disorder diagnoses and those from other methods and sources. Clinical Psychology: Science and Practice, 22(1), 1-19. doi:10.1111/cpsp.12088

Samuel, D. B., Ansell, E. B., Hopwood, C. J., Morey, L. C., Markowitz, J. C., Skodol, A. E., \& Grilo, C. M. (2010). The impact of NEO PI-R gender norms on the assessment of personality disorder profiles. Psychological Assessment, 22(3), 539-545. doi:10.1037/a0019580

Samuel, D. B., \& Gore, W. L. (2012). Maladaptive variants of conscientiousness and agreeableness. Journal of Personality, 80(6), 1669-1696. doi:10.1111/i.1467-6494.2012.00770.x

Samuel, D. B., \& Widiger, T. A. (2004). Clinicians' personality descriptions of prototypic personality disorders. Journal of Personality Disorders, 18(3: Special issue), 286-308. doi:10.1521/pedi.18.3.286.35446

Samuel, D. B., \& Widiger, T. A. (2008). A meta-analytic review of the relationships between the five-factor model and DSM-IV-TR personality disorders: A facet level analysis. Clinical Psychology Review, 28(8), 13261342. doi:10.1016/i.cpr.2008.07.002

Samuel, D. B., \& Widiger, T. A. (2009). Comparative gender biases in models of personality disorder. Personality and Mental Health, 3(1), 12-25. doi:10.1002/pmh.61

Samuel, D. B., \& Widiger, T. A. (2011). Conscientiousness and obsessivecompulsive personality disorder. Personality Disorders: Theory, Research, and Treatment, 2(3), 161-174. doi:10.1037/a0021216
Saulsman, L. M., \& Page, A. C. (2004). The five-factor model and personality disorder empirical literature: A meta-analytic review. Clinical Psychology Review, 23(8), 1055-1085. doi:10.1016/j.cpr.2002.09.001

Trull, T. J. (2012). The five-factor model of personality disorder and DSM5. Journal of Personality, 80(6), 1697-1720. doi:10.1111/j.14676494.2012.00771.x

Trull, T. J., \& Durrett, C. A. (2005). Categorical and dimensional models of personality disorder. Annual Review of Clinical Psychology, 1, 355-380. doi:10.1146/annurev.clinpsy.1.102803.144009

Trull, T. J., \& Widiger, T. A. (2013). Dimensional models of personality: the five-factor model and the DSM-5. Dialogues in Clinical Neuroscience, $15(2), 135-146$

Widiger, T. A. (2007). Dimensional models of personality disorder. World Psychiatry, 6(2), 15-19.

Widiger, T. A. (2015). Assessment of DSM-5 Personality Disorder. Journal of Personality Assessment, 97(5), 456-466. doi:10.1080/00223891.2015.1041142

Widiger, T. A., \& Boyd, S. (2009). Personality disorder assessment instruments. In J. Butcher (Ed.), Oxford Handbook of Personality Assessment (3rd ed., pp. 336-363). New York, NY: Oxford University Press.

Widiger, T. A., \& Costa, P. T. (2012). Integrating normal and abnormal personality structure: The five-factor model. Journal of Personality, $80(6)$, 1471-1506. doi:10.1111/j.1467-6494.2012.00776.x

Widiger, T. A., \& Costa, P. T. (Eds.). (2013). Personality disorders and the fivefactor model of personality (3rd ed.). Washington, DC: American Psychological Association.

Widiger, T. A., \& Mullins-Sweatt, S. N. (2009). Five-factor model of personality disorder: A proposal for DSM-V. Annual Review of Clinical Psychology, 5, 197-220. doi:10.1146/annurev.clinpsy.032408.153542

Widiger, T. A., \& Mullins-Sweatt, S. N. (2010). Clinical utility of a dimensional model of personality disorder. Professional Psychology: Research and Practice, 41(6), 488-494. doi:10.1037/a0021694

Widiger, T. A., \& Presnall, J. R. (2013). Clinical application of the fivefactor model. Journal of Personality, 81(6), 515-527. doi:10.1111/jopy.12004

Widiger, T. A., \& Samuel, D. B. (2005). Diagnostic categories or dimensions? A question for the Diagnostic and Statistical Manual of Mental Disorders-Fifth edition. Journal of Abnormal Psychology, 114(4), 494-504. doi:10.1037/0021-843X.114.4.494

Widiger, T. A., \& Simonsen, E. (2005). Alternative dimensional models of personality disorder: Finding a common ground. Journal of Personalit Disorders, 19(2), 110-130. doi:10.1521/pedi.19.2.110.62628

Widiger, T. A., \& Trull, T. J. (2007). Plate tectonics in the classification of personality disorder: Shifting to a dimensional model. American Psycbologist, 62(2), 71-83. doi:10.1037/0003-066X.62.2.71

Widiger, T. A., Trull, T. J., Clarkin, J. F., Sanderson, C., \& Costa, P. T. (1994). A description of the DSM-III-R and DSM-IV personality disorders with the five-factor model of personality. In P. T. Costa \& T. A Widiger (Eds.), Personality Disorders and the Five-Factor Model of Personality (pp. 41-56). Washington, DC: American Psychological Association.

Widiger, T. A., Trull, T. J., Clarkin, J. F., Sanderson, C., \& Costa, P. T. (2002). A description of the DSM-IV personality disorders with the five-factor model of personality. In P. T. Costa \& T. A. Widiger (Eds.), Personality Disorders and the Five-Factor Model of Personality (2nd ed., pp. 8999). Washington, DC: American Psychological Association.

Wille, B, De Fruyt, F, \& De Clercq, B. (2013). Expanding and reconceptualizing aberrant personality at work: Validity of five-factor model aberrant personality tendencies to predict career outcomes. Personnel Psycbology, 66(1), 173-223. doi:10.1111/peps.12016

(Article received: 18-03-2017; revised: 20-04-2017; accepted: 02-05-2017) 
Appendix. Additive counts for the calculation of personality disorders from the facets of NEO-PI-R.

PD

Paranoid

Schizoid

Schizotypal

Antisocial

Borderline

Histrionic

Narcissistic

Avoidant

Dependent

Obsessive Facets

$\mathrm{N} 2+\mathrm{E} 1 r+\mathrm{E} 2 \mathrm{r}+\mathrm{O} 4 \mathrm{r}+\mathrm{O} 6 \mathrm{r}+\mathrm{A} 1 \mathrm{r}+\mathrm{A} 2 \mathrm{r}+\mathrm{A} 3 \mathrm{r}+\mathrm{A} 4 \mathrm{r}+\mathrm{A} 6 \mathrm{r}$

$\mathrm{E} 1 r+\mathrm{E} 2 r+\mathrm{E} 3 r+\mathrm{E} 4 r+\mathrm{E} 5 r+\mathrm{E} 6 r+\mathrm{O} 3 r+\mathrm{O} 4 r$

$\mathrm{N} 1+\mathrm{N} 4+\mathrm{E} 1 \mathrm{r}+\mathrm{E} 2 \mathrm{r}+\mathrm{E} 6 \mathrm{r}+\mathrm{O} 5+\mathrm{C} 2 \mathrm{r}$

$\mathrm{N} 1 \mathrm{r}+\mathrm{N} 2+\mathrm{N} 4 \mathrm{r}+\mathrm{N} 5+\mathrm{E} 3+\mathrm{E} 4+\mathrm{E} 5+\mathrm{O} 4+\mathrm{A} 1 \mathrm{r}+\mathrm{A} 2 \mathrm{r}+\mathrm{A} 3 \mathrm{r}+\mathrm{A} 4 \mathrm{r}+\mathrm{A} 5 \mathrm{r}+\mathrm{A} 6 \mathrm{r}+\mathrm{C} 3 \mathrm{r}+\mathrm{C} 5 \mathrm{r}+\mathrm{C} 6 \mathrm{r}$

$\mathrm{N} 1+\mathrm{N} 2+\mathrm{N} 3+\mathrm{N} 5+\mathrm{N} 6+\mathrm{O} 3+\mathrm{O} 4+\mathrm{A} 4 \mathrm{r}+\mathrm{C} 6 \mathrm{r}$

$\mathrm{N} 4 \mathrm{r}+\mathrm{N} 5+\mathrm{E} 2+\mathrm{E} 4+\mathrm{E} 5+\mathrm{E} 6+\mathrm{O} 1+\mathrm{O} 3+\mathrm{O} 4+\mathrm{A} 1+\mathrm{C} 5 \mathrm{r}+\mathrm{C} 6 \mathrm{r}$

$\mathrm{N} 2+\mathrm{N} 4 \mathrm{r}+\mathrm{E} 1 \mathrm{r}+\mathrm{E} 3+\mathrm{E} 5+\mathrm{O} 3 \mathrm{r}+\mathrm{O} 4+\mathrm{A} 1 \mathrm{r}+\mathrm{A} 2 \mathrm{r}+\mathrm{A} 3 \mathrm{r}+\mathrm{A} 4 \mathrm{r}+\mathrm{A} 5 \mathrm{r}+\mathrm{A} 6 \mathrm{r}$

$\mathrm{N} 1+\mathrm{N} 4+\mathrm{N} 5 \mathrm{r}+\mathrm{N} 6+\mathrm{E} 2 \mathrm{r}+\mathrm{E} 3 \mathrm{r}+\mathrm{E} 5 \mathrm{r}+\mathrm{E} 6 \mathrm{r}+\mathrm{O} 4 \mathrm{r}+\mathrm{A} 5$

$\mathrm{N} 1+\mathrm{N} 3+\mathrm{N} 4+\mathrm{N} 6+\mathrm{C} 1 \mathrm{r}+\mathrm{C} 5 \mathrm{r}$

$\mathrm{N} 1+\mathrm{N} 5 \mathrm{r}+\mathrm{E} 5 \mathrm{r}+\mathrm{O} 3 \mathrm{r}+\mathrm{O} 4 \mathrm{r}+\mathrm{O} 5 \mathrm{r}+\mathrm{O} 6 \mathrm{r}+\mathrm{C} 1+\mathrm{C} 2+\mathrm{C} 3+\mathrm{C} 4+\mathrm{C} 5+\mathrm{C} 6$

Note: PD prototypes: Lynam \& Widiger (2001), except Dependent PD (Miller \& Lynam, 2008). N1 = Anxiety; N2 = Angry Hostility; N3 = Depression; N4

= Self-Consciousness; N5 = Impulsiveness; N6 = Vulnerability; E1 = Warmth; E2 = Gregariousness; E3 = Assertiveness; E4 = Activity; E5 = ExcitementSeeking; E6 = Positive Emotions; O1 = Fantasy; O3 = Feelings; O4 = Actions; O5 = Ideas; O6 = Values; A1 = Trust; A2 = Straightforwardness; A3 = Altruism; A4 = Compliance; A5 = Modesty; A6 = Tender-Mindedness; $\mathrm{C} 1=$ Competence; $\mathrm{C} 2=$ Order; $\mathrm{C} 3=$ Dutifulness; $\mathrm{C} 4=\mathrm{Achievement}$ Striving; $\mathrm{C} 5=$ Self-Discipline; $\mathrm{C} 6=$ Deliberation. $\mathrm{r}=$ the value of this facet in NEO-PI-R should be reverse scored before summing it into the count so that all facets are scored in the same maladaptive direction. 